\title{
Isolation and characterization of human breast tumor-derived endothelial cells
}

\author{
CRISTINA GRANGE ${ }^{1 *}$, BENEDETTA BUSSOLATI ${ }^{1 *}$, STEFANIA BRUNO $^{1}$, \\ VALENTINA FONSATO $^{1}$, ANNA SAPINO ${ }^{2}$ and GIOVANNI CAMUSSI ${ }^{1}$ \\ ${ }^{1}$ Department of Internal Medicine and Research Center for Experimental Medicine and \\ ${ }^{2}$ Department of Biomedical Sciences and Human Oncology, University of Torino, Torino, Italy
}

Received June 24, 2005; Accepted August 2, 2005

\begin{abstract}
Increasing evidence indicates that tumor-derived endothelial cells (TEC) possess a distinct and unique phenotype in respect to normal endothelial cells and may be able to acquire resistance to drugs. However, few functional studies are available on cultured TEC. In the present study, we obtained TEC from human breast carcinomas and, to dispel the possibility of contaminating tumor cells, we established six different clones that we characterized at a functional level. Breast TEC cell lines and clones did not undergo normal cell senescence in culture and showed constant expression of markers of endothelial activation and angiogenesis. These cells showed increased apoptosis resistance to vincristine and doxorubicin and increased random cell motility, as compared to normal micro-endothelial cells. In addition, breast TEC, at variance to normal endothelial cells, were able to grow and to organize in the absence of serum in capillary-like structures on Matrigel that persisted up to one week. These functional characteristics of breast TEC may be relevant for tumor angiogenesis and may indicate an increased pro-angiogenic activity of endothelial cells within the tumor. Moreover, our data suggest that TEC might be more appropriate for screening antiangiogenic drugs than normal endothelial cells.
\end{abstract}

\section{Introduction}

The process of angiogenesis leads to the formation of new vessels and requires a critical contribution of endothelial cells (1). The so-called 'switch' of the tumor to an angiogenic phenotype is considered a hallmark of the malignant process

Correspondence to: Dr Giovanni Camussi, Department of Internal Medicine, University of Torino, Corso Dogliotti 14, 10126 Torino, Italy

E-mail: giovanni.camussi@unito.it

${ }^{*}$ Contributed equally

Key words: tumor-derived endothelial cells, angiogenesis, breast carcinoma, apoptosis resistance
(2). In solid tumors, angiogenesis is important for supporting the rapid growth of the tumor beyond 1-2 mm in diameter and serves as the gatekeeper for tumor cell escape and entry into the circulation $(3,4)$. It is well established that blood vessels in tumors differ from normal vessels by altered morphology, blood flow and permeability and by abnormalities in pericytes and in basement membrane (5-9). Evidence indicates that tumor-derived endothelial cells (TEC) express surface molecules, such as the receptors for the adhesion to matrix and to circulating leukocytes and for angiogenic growth factors that are absent or barely detectable in established blood vessels (10). The structural and molecular diversity of tumor-associated vasculature provides a basis for the development of targeted diagnostics and therapeutics (11-13). However, an emerging concept in tumor therapy is the unresponsiveness of human tumors to anti-angiogenic therapy (14). TEC were reported to possess distinct and unique phenotypes (15) and to be able to acquire resistance to drugs (16). In addition, we previously demonstrated that TEC derived from human renal carcinomas are different from normal vessels at the molecular and functional levels (17). It has been shown that TEC derived from different tumors are genetically unstable (18) challenging the established concept that TEC are susceptible to chemotherapeutic therapy due to their genetic stability. However, functional studies on cultured TEC are limited to human renal carcinoma-derived TEC (17) and murine lung carcinoma-derived TEC (19). The aim of the present study was to establish and characterize at a functional level TEC obtained from human breast carcinomas.

\section{Materials and methods}

Reagents and antibodies. ECAF, DMEM, PBS tablets, bovine serum albumin fraction $\mathrm{V}$ (tested for not more than $1 \mathrm{ng}$ endotoxin per mg) were purchased from Sigma Chemical Company (St. Louis, MO). EBM complete medium was obtained from Cambrex Bioscience (Baltimore, MD), fetal calf serum (FCS) and RPMI were from EuroClone Ltd. (Wetherby West Yorkshire, UK). All the antibodies were against human antigens. Anti-ICAM-1, anti- $\alpha \mathrm{V} \beta 3$ and $\beta 1 \mathrm{mAbs}$ were from B\&D Pharmingen (San Jose, CA), FITC-labeled anti-CD105 $\mathrm{mAb}$ was from Serotec (Raleigh, NC), PE-labeled antiCD146 mAb was from Cabru (Milan, Italy), PE-labeled antiKDR, anti-vascular endothelial growth factor-receptor 
Table I. Expression of endothelial markers by six different clones of B-TEC.

\begin{tabular}{|c|c|c|c|c|c|c|}
\hline \multirow{3}{*}{ CD105 } & \multirow{3}{*}{$\begin{array}{l}\text { B-TEC/A } \\
90 \backslash 87^{\mathrm{a}}\end{array}$} & \multirow{3}{*}{$\begin{array}{c}\text { B-TEC/B } \\
13 \backslash 83^{\mathrm{a}}\end{array}$} & \multirow{3}{*}{$\begin{array}{r}\text { B-TEC/C } \\
\text { Mean fluorescence } \\
45 \backslash 80^{\mathrm{a}}\end{array}$} & \multirow{3}{*}{$\begin{array}{l}\text { B-TEC/E } \\
\text { ty\\
% positiv } \\
7 \backslash 62^{\mathrm{a}}\end{array}$} & \multirow{3}{*}{$\begin{array}{l}\text { B-TEC/F } \\
84 \backslash 90^{\mathrm{a}}\end{array}$} & \multirow{3}{*}{$\frac{\mathrm{B}-\mathrm{TEC} / \mathrm{G}}{13 \backslash 61^{\mathrm{a}}}$} \\
\hline & & & & & & \\
\hline & & & & & & \\
\hline CD31 & $3 \backslash 3$ & $5 \backslash 15^{\mathrm{a}}$ & $10 \backslash 10^{\mathrm{a}}$ & $6 \backslash 22^{a}$ & $5 \backslash 48^{a}$ & $7 \backslash 46^{a}$ \\
\hline MUC18 & $90 \backslash 98^{a}$ & $119 \backslash 97^{\mathrm{a}}$ & $61 \backslash 90^{\mathrm{a}}$ & $38 \backslash 93^{\mathrm{a}}$ & $87 \backslash 96^{\mathrm{a}}$ & $45 \backslash 92^{a}$ \\
\hline CD44 & $72 \backslash 98^{a}$ & $83 \backslash 97^{a}$ & $76 \backslash 93^{a}$ & $75 \backslash 92^{a}$ & $86 \backslash 91^{\mathrm{a}}$ & $54 \backslash 46^{\mathrm{a}}$ \\
\hline KDR & $7 \backslash 33^{a}$ & $6 \backslash 13^{a}$ & $9 \backslash 17^{a}$ & $5 \backslash 4$ & $6 \backslash 16^{a}$ & $3 \backslash 6$ \\
\hline VEGFR3 & $10 \backslash 51^{\mathrm{a}}$ & $14 \backslash 44^{\mathrm{a}}$ & $10 \backslash 13^{\mathrm{a}}$ & $11 \backslash 15^{\mathrm{a}}$ & $80 \backslash 38^{\mathrm{a}}$ & $14 \backslash 48^{a}$ \\
\hline$\beta 1$ & $41 \backslash 91^{\mathrm{a}}$ & $110 \backslash 93^{a}$ & $45 \backslash 76^{\mathrm{a}}$ & $97 \backslash 99^{a}$ & $58 \backslash 90^{\mathrm{a}}$ & ND \\
\hline ICAM & ND & $97 \backslash 89^{a}$ & $145 \backslash 98^{\mathrm{a}}$ & $79 \backslash 98^{a}$ & $61 \backslash 88^{a}$ & ND \\
\hline
\end{tabular}

${ }^{\mathrm{a}} \mathrm{p}<0.001$ versus isotypic control, as evaluated using the Kolmogorov-Smirnov statistical analysis. Marker expression was evaluated by FACS analysis and results expressed as mean fluorescence intensity and as percentage of positive cells. Results are the mean of three different experiments. ND, not done.

(VEGF-R)-3 mAbs were from RD System (Minneapolis, MN). The anti-vascular endothelial cadherin was kindly provided by Professor G. Tarone (University of Torino, Italy). FITClabeled anti-CD31 was purchased from Euroclone and FITClabeled anti-CD44 was obtained from Sigma Chemical Company. Secondary PE-labeled anti-rat IgG was from Caltag Laboratories (Burlingame, CA); secondary PE-labeled antimouse IgG was purchased by Dako (Milan). Polyclonal Ab against human von Willebrand factor (vWF) was obtained from Santa Cruz Biotechnology (Santa Cruz, CA). Vincristine, doxorubicin, cell dissociation solution, trypsin-EDTA solution (10X) and collagenase IV were obtained from Sigma. Gelatin for coating of dishes was from Difco Laboratories (Detroit, USA).

Endothelial cell lines. Tumor-derived endothelial cells were obtained from breast carcinomas (three ductal and a tubulolobular infiltrating breast carcinomas) or from normal surrounding breast tissue. Specimens were finely minced with scissors and then digested by incubation for $1 \mathrm{~h}$ at $37^{\circ} \mathrm{C}$ in DMEM containing collagenase IV (Sigma). After washings in medium plus $10 \%$ FCS, the cell suspension was forced through a graded series of meshes to separate the cell components from stroma and aggregates. Endothelial cells were isolated from cells suspension using anti-CD105 Ab coupled to magnetic beads, by magnetic cell-sorting using the MACS system (Miltenyi Biotech, Auburn, CA). Cells were grown in complete EBM medium (Cambrex) supplemented with $10 \%$ FCS, $50 \mathrm{U} / \mathrm{ml}$ penicillin, and $50 \mu \mathrm{g} / \mathrm{ml}$ streptomycin as previously described (17). HMEC were obtained from derma using anti-CD31 Ab coupled to magnetic beads, by magnetic cell-sorting using the MACS system (Miltenyi Biotech), as previously described (20).

Cell-cloning was performed by the limiting dilution technique and single cells were deposited in 96-well plates in the presence of culture medium.
Immunofluorescence studies. For cytofluorimetric analysis, cells were detached from plates with a non-enzymatic cell dissociation solution (Sigma), washed in PBS containing 2\% heat-inactivated human serum and incubated for another $15 \mathrm{~min}$ with whole heat-inactivated human serum to block remaining non-specific sites. Cells were then incubated for $30 \mathrm{~min}$ at $4^{\circ} \mathrm{C}$ with the appropriate $\mathrm{Ab}$ or with the irrelevant control in PBS containing $2 \%$ heat-inactivated human serum. Where needed, cells were stained by the addition of PE-conjugated goat anti-mouse or goat anti-rabbit Abs, and incubated for further $30 \mathrm{~min}$ at $4^{\circ} \mathrm{C}$. Cells were analysed on a FACS (Becton Dickinson, Mountain View, CA). Cells (10000) were analysed in each experimental point.

Immunofluorescence was also performed on paraffinembedded sections after antigen retrieval with three cycles of $15 \mathrm{sec}$ microwave treatment. The sections were washed in PBS and incubated with the Abs for $2 \mathrm{~h}$ at room temperature. When needed a second step, FITC or PE conjugated Ab was added for $30 \mathrm{~min}$ at room temperature. Control experiments included incubation with isotypic control mouse IgG. Three nonsequential sections were examined for each specimen.

Assessment of apoptosis. Apoptosis was evaluated using the TUNEL assay (ApopTag Oncor, Gaithersburg, MD). After treatment with vincristine or doxorubicin for $24 \mathrm{~h}$, cells were suspended in PBS, fixed in $1 \%$ paraformaldehyde in PBS $\mathrm{pH} 7.4$ for $15 \mathrm{~min}$ at $4^{\circ} \mathrm{C}$, and then in pre-cooled ethanol-acetic acid $2: 1$ for $5 \mathrm{~min}$ at $-20^{\circ} \mathrm{C}$. Cells were treated with terminal deoxynucleotidyl transferase enzyme and incubated in a humidified chamber at $37^{\circ} \mathrm{C}$ for $1 \mathrm{~h}$, and then with FITCconjugated anti-digoxigenin for $30 \mathrm{~min}$ at room temperature. After washings, samples were mounted in medium containing $1 \mu \mathrm{g} / \mathrm{ml}$ of propidium iodide and the cells analysed by immunofluorescence. Results are expressed as percentage of green fluorescence emitting cells (apoptotic cells) versus red fluorescence emitting cells (total cells). 

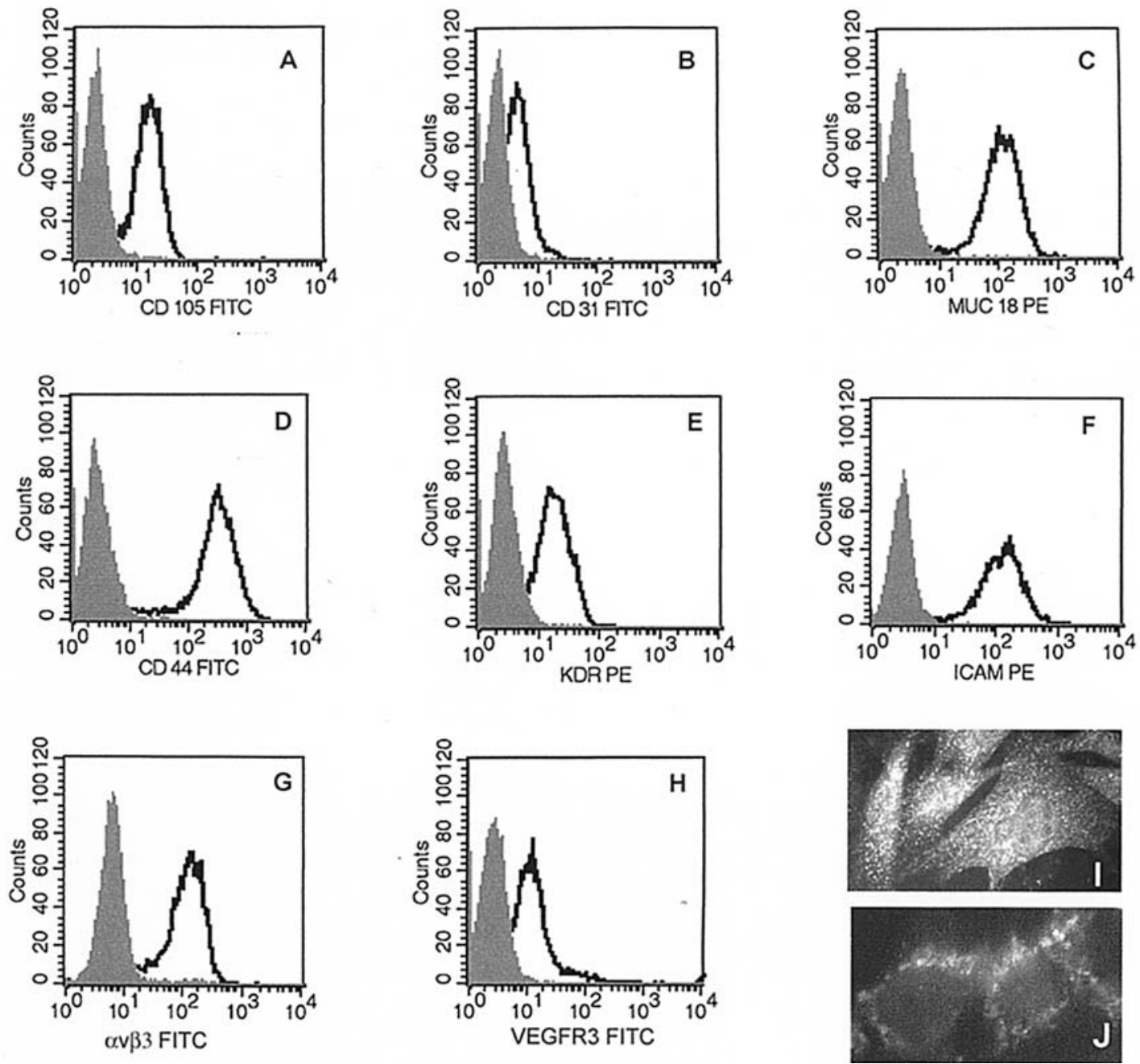

Figure 1. Characterization of B-TEC. Panels A-H: Representative flow cytometric analysis of B-TEC-01 cells showing expression (dark line) of the endothelial markers CD105 (A), CD31 (B), Muc-18 (C), CD44 (D), KDR (E), ICAM (F), avB3 (G), VEGFR3 (H). The grey area is the isotypic control. Panel I: micrograph representative of vWF expression. Panel J: micrograph representative of vascular endothelial-cadherin (I and J: original magnification: $\mathrm{x} 400$ ). Four B-TEC cell preparations (01-04) were analysed with similar results.

In vitro cell migration. Cell migration of quiescent adherent B-TEC (10 cell/well in RPMI plus $0.25 \%$ BSA) was studied over $12 \mathrm{~h}$ period under a Nikon Diaphot-inverted microscope with a 10X phase-contrast objective, as previously described (21). Cells were kept in an attached, hermetically sealed plexiglass Nikon NP-2 incubator at $37^{\circ} \mathrm{C}$. Cell migration was recorded using a JVC-1CCD video camera. Image analysis was performed with a MicroImage analysis system (Cast Imaging srl, Venice, Italy) and an IBM-compatible system equipped with a video card (Targa 2000, Truevision, Santa Clara, CA). Image analysis was performed by digital saving of images at $30 \mathrm{~min}$ interval. Migration tracks were generated by marking the position of nucleus of individual cells on each image. The net migratory speed 'velocity straight line' was calculated by the MicroImage software based on the straight line distance between the starting and ending points divided by the time of observation. Migration of at least 30 cells was analyzed for each experimental condition. Values are given as mean \pm SD. Cell division did not start to any significant degree during the experiments.

Tube formation. In vitro formation of tubular structures was studied on growth factor-reduced Matrigel in a 24-well plate.
Cells $\left(3 \times 10^{4}\right.$ cells/well) were seeded onto Matrigel-coated wells in RPMI medium containing $0.25 \%$ BSA and 0 or $5 \%$ FCS. Cells were periodically observed with a Nikon inverted microscope and experimental results recorded. Image analysis was performed with the MicroImage analysis system (Cast Imaging srl).

\section{Results}

Endothelial cells were purified by breast carcinomas using magnetic beads coupled to endoglin (CD105). CD105 was chosen because previous investigations with histochemical staining showed overexpression of this marker in blood vessels at the site of neovascularization in mammary tumors (22). Immunohistochemical evaluation of the tumor tissue showed that this marker was expressed by vascular structures and not by the tumor cells (not shown). Four cell preparations of breastTEC (B-TEC 01-04) were obtained from different tumors. FACS analysis showed CD105 expression in $92 \pm 3.4 \%$ of immunosorted cells obtained from the tumor. The immunosorted cells were characterized by cytofluorimetric analysis on the basis of positive expression of a panel of endothelial markers (10), such as CD105, CD31, Muc-18 (CD146), 
A

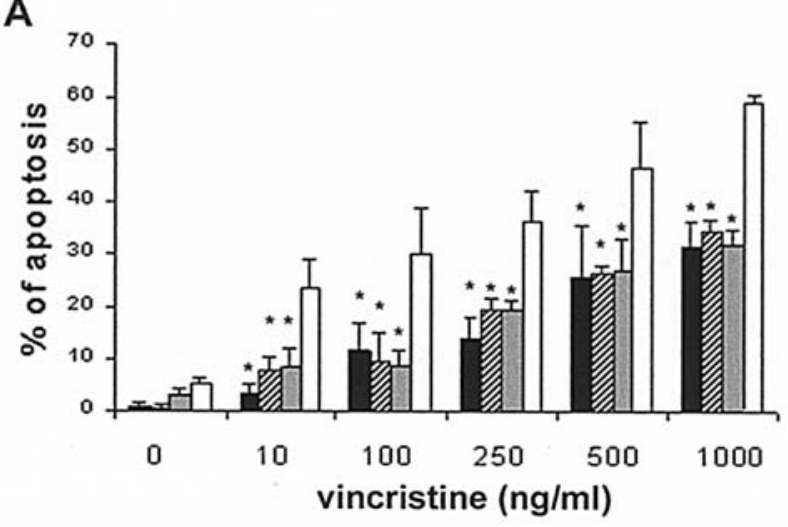

B

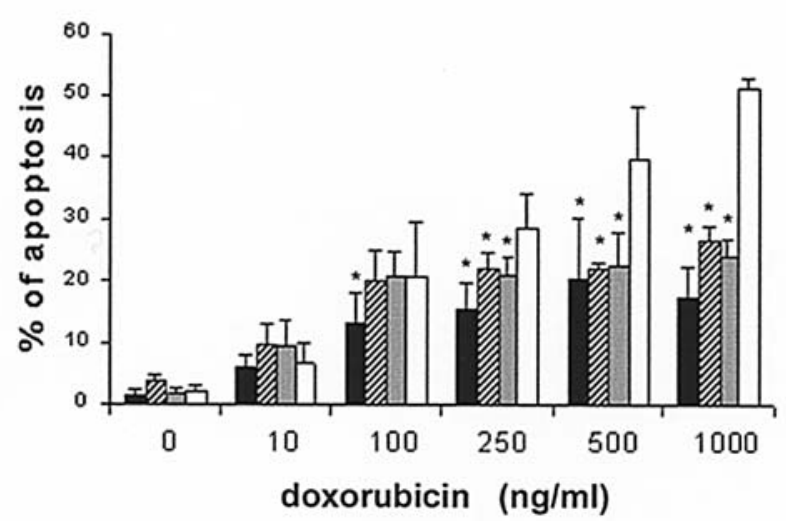

C

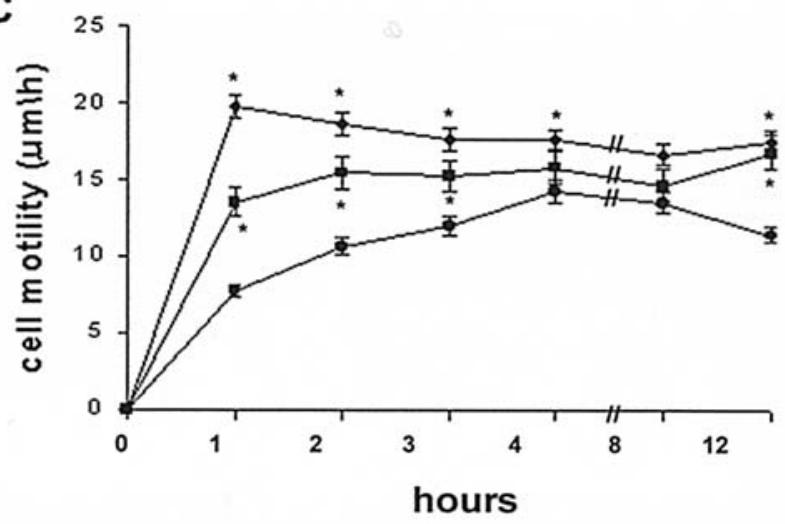

D



Figure 2. Apoptosis resistance, motility and tubulogenesis of B-TEC clones in respect to normal HMEC. Panel A and B: Increased apoptosis of normal HMEC (white column) in respect to 2 different clones of B-TEC (clones A and F; dark and hatched columns, respectively) and to B-TEC01 cell line (gray columns) after incubation with different doses of vincristine (A) or doxorubicin (B) for $24 \mathrm{~h}$. Apoptosis was detected as TUNEL assay. Data are the mean \pm SD of six different experiments performed in duplicate. Panel C: Increased random cell motility of two different clones of B-TEC (clones B and G, a and $\bullet$, respectively) in respect to HMEC ( $\mathbf{\Delta})$. Motility was measured by time-lapse analysis (Material and methods). Results are expressed as mean \pm SD of three separate experiments. Panel D: In vitro angiogenesis of B-TEC clones. B-TEC and normal HMEC (3x $10^{4}$ cells/well) were plated on growth factor-reduced Matrigel in RPMI containing 5\% FCS and observed at different times. An increase in tube formation was observed in different clones of B-TEC (clones A, C and F; dark, hatched and gray columns respectively) in respect to HMEC (white columns). Data are expressed as the mean \pm SEM of the tube lengths evaluated in five different fields at magnification x20 in duplicate wells of three different experiments. ANOVA with Dunnet's multicomparison test was performed between B-TEC clones versus HMEC $\left({ }^{*} \mathrm{p}<0.05\right)$.

CD44 and VEGFR2 (KDR) (Fig. 1A-E) and by expression of vWF (Fig. 1I) but not cytokeratin (not shown) by immunofluorescence on permeabilized cells. B-TEC also expressed the adhesion molecules ICAM-I and $\alpha \mathrm{v} \beta 3$ (Fig. 1F and G) and B1 integrin (not shown) (23). In addition, B-TEC expressed vascular endothelial-cadherin (Fig. 1J) and the VEGFR-3, the lymphatic-associated VEGF receptor, which is also expressed by renal TEC (17) and by vascular tumors $(24,25)$ (Fig. 1H). To dispel the possibility that contaminating tumor cells may influence our results, we cloned the B-TEC01 cell line by limited dilution and we obtained and characterized six different clones. All these clones confirmed the same pattern of endothelial marker expression as the progenitor B-TEC01 cell line (Table I). The level of expression of endothelial markers was tested every five-six culture passages and remained constant during cell culture.

To determine the behavior of B-TEC, cell resistance to apoptosis, migration and organization onto Matrigel of B-TEC lines and clones was compared with that of normal HMEC derived from derma. At variance of normal HMEC, B-TEC did not show senescence in culture and were able to grow for $>50$ passages. In addition, B-TEC cell lines and clones showed a higher resistance to apoptosis induced by the chemotherapeutic drugs vincristine and doxorubicin as compared to HMEC (Fig. 2A and B). Furthermore, B-TEC showed an increased spontaneous random motility in respect to HMEC, as detected by time-lapse cinematography (Fig. 2C).

As HMEC, B-TEC showed the ability to make tubes when plated onto Matrigel with serum. At $6 \mathrm{~h}$, under the condition of low level of serum (RPMI and 5\% FCS), B-TEC clones showed enhanced formation of tubes in respect to HMEC. Similar results were obtained with B-TEC lines (not shown). This difference was also evident after 24 or 48 h (Figs. 2D and 3). At variance to HMEC, that undergo apoptosis when plated onto Matrigel in the absence of serum (17), B-TEC were able to form tubes also in $0 \%$ serum. In addition, the structures formed by B-TEC cell lines and clones persisted several days without serum (Fig. 3).

\section{Discussion}

In the present study we isolated, cloned and characterized the phenotypic and functional properties of endothelial cells derived from breast carcinomas. These cells did not undergo 
A
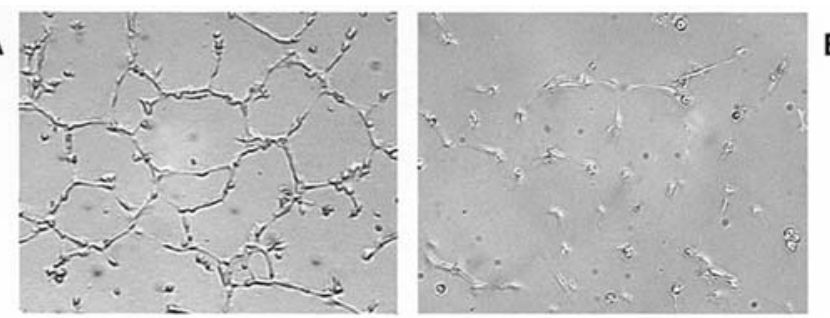

Figure 3. Tube formation of B-TEC/E in the absence of serum. Representative micrograph of the tubes formed by B-TEC01/E in the absence of serum and maintained up to seven days (A). HMEC did not form tubes in the absence of serum (B). Magnification of x150.

normal cell senescence in culture and showed increased apoptosis resistance, motility and pro-angiogenic properties.

Tumor angiogenesis is a critical process in the development and progression of cancer and it is currently regarded as a potential therapeutic target. This therapeutic approach is based on the concept that endothelial cells are genetically stable and therefore they should not develop drug resistance. However, it has become clear from preliminary trials that a number of anti-angiogenic drugs or strategies can lose their activity over time $(26,27)$. Several potential mechanisms have been evocated, such as the great redundancy of tumor-secreted angiogenic growth factors and the anti-apoptotic properties of TEC dependent either on direct tumor-endothelial cell interaction or on epigenetic changes occurring after persistent cell activation (28). Recently, it has been reported that tumor endothelium has, at the molecular level, distinct characteristics from normal endothelial cells (15) and may be resistant to apoptosis $(14,29)$. In addition, it has been shown that human TEC from melanoma and liposarcoma are resistant to apoptosis induced by etoposide (18) and that human TEC from renal carcinoma are resistant to vincristine (17). In the present study, we report that human TEC derived from breast carcinoma exhibited altered survival and showed resistance to apoptosis induced by vincristine or doxorubicin. These data suggest that the reported drug resistance of TEC is not a tumor type limited characteristic, but rather is a more general phenomenon.

We also found that B-TEC displayed an enhanced motility in respect to normal HMEC. In addition, B-TEC, at variance to normal endothelial cells, were able to organize, in the absence of serum, in capillary-like structures on Matrigel that persisted up to one week. These functional characteristics of B-TEC may be relevant for tumor angiogenesis and may indicate an increased pro-angiogenic activity of these cells.

Moreover, these characteristics of B-TEC were maintained in cell culture over time and were independent of the contamination by tumor cells as they were present in clones derived from a single cell. These data suggest that B-TEC underwent a persistent change in the normal endothelial phenotype. This could be due to genetic mutations, as suggested by the reported genetic instability of TEC (18) or to viral oncogenes, as indicated by data on HHV-8-infected endothelial cells in Kaposi's sarcoma (30). In addition, a multistep process involving mutations of both the epithelial and the mesenchymal compartment has been described in breast carcinoma (28), suggesting a possible reciprocal interaction between tumor and surrounding cells. Alternatively, it can be speculated that TEC may originate from endothelial precursors, known to display an enhanced angiogenic potential (31). Finally, it has been proposed that tumor cells in breast carcinomas may dedifferentiate and participate in tumor angiogenesis by vascular mimicry (32).

In conclusion, the results of the present study show that endothelial cells derived from breast carcinoma maintained a pro-angiogenic phenotype and displayed resistance to apoptosis independent of the presence of tumor cells. Moreover, our data provide a rationale for the observed drug resistance of TEC and suggest that TEC might be more appropriate for screening antiangiogenic drugs than normal endothelial cells.

\section{Acknowledgements}

This study was supported by the Associazione Italiana per la Ricerca sul Cancro (AIRC), by Istituto Superiore di Sanità (Targeted Project AIDS), by the National Research Council (CNR), by the Italian Ministry of University and Research (MIUR) FIRB project (RBNE01HRS5-001) and COFIN 01, by the Italian Ministry of Health (Ricerca Finalizzata 02) and by Special Project Oncology, Compagnia di S. Paolo/FIRMS to G.C.

\section{References}

1. Carmeliet P: Mechanisms of angiogenesis and arteriogenesis. Nat Med 3: 389-395, 2000.

2. Hicklin DJ and Ellis LM: Role of the vascular endothelial growth factor pathway in tumor growth and angiogenesis. J Clin Oncol 23: 1011-1027, 2005.

3. Folkman J: Angiogenesis in cancer, vascular, rheumatoid and other disease. Nat Med 1: 27-31, 1995.

4. Folkman J: Seminars in medicine of the Beth Israel Hospital, Boston. Clinical applications of research on angiogenesis. N Engl J Med 26: 1757-1763, 1995.

5. Jain RK: Molecular regulation of vessel maturation. Nat Med 9: 685-693, 2003

6. Mollica F, Jain RK and Netti PA: A model for temporal heterogeneities of tumor blood flow. Microvasc Res 65: 56-60, 2003.

7. McDonald DM and Baluk P: Significance of blood vessel leakiness in cancer. Cancer Res 62: 5381-5385, 2002.

8. Baluk P, Morikawa S, Haskell A, Mancuso M and McDonald DM: Abnormalities of basement membrane on blood vessels and endothelial sprouts in tumors. Am J Pathol 163: 1801-1815, 2003.

9. Morikawa S, Baluk P, Kaidoh T, Haskell A, Jain RK and McDonald DM: Abnormalities in pericytes on blood vessels and endothelial sprouts in tumors. Am J Pathol 160: 985-1000, 2002.

10. Garlanda $\mathrm{C}$ and Dejana E: Heterogeneity of endothelial cells. Specific markers. Arterioscler Thromb Vasc Biol 17: 1193-1202, 1997.

11. Pasqualini R: In vivo phage display and vascular heterogeneity: implication for targeted medicine. Curr Opin Chem Biol 6: 399-404, 2002.

12. Nathwani AC: Antiangiogenetic gene therapy for cancer treatment. Curr Hematol Rep 3: 267-273, 2004.

13. Miller KD, Sweeney CJ and Sledge GW Jr: Redefining the target: chemotherapeutics as antiangiogenics. J Clin Oncol 19: 1195-1206, 2001.

14. Iwahana M, Utoguchi N, Mayumi T, Goryo M and Okada K: Drug resistance and P-glycoprotein expression in endothelial cells of newly formed capillaries induced by tumors. Anticancer Res 18: 2977-2980, 1998.

15. St. Croix B, Rago C, Velculescu V, Traverso G, Romans KE, Montgomery E, Lal A, Riggins GJ, Lengauer C, Vogelstein B and Kinzler KW: Genes expressed in human tumor endothelium. Science 289: 1197-1202, 2000.

16. Jung YD, Ahmad SA, Akagi Y, Takahashi Y, Liu W, Reinmuth N, Shaheen RM, Fan F and Ellis LM: Role of the tumor microenvironment in mediating response to anti-angiogenic therapy. Cancer Metastasis Rev 19: 147-157, 2000. 
17. Bussolati B, Deambrosis I, Russo S, Deregibus MC and Camussi G: Altered angiogenesis and survival in endothelial cells derived from renal carcinoma. FASEB J 17: 1159-1161, 2003.

18. Hida K, Hida Y, Amin DN, Flint AF, Panigrahy D, Morton CC and Klagsbrun M: Tumor-associated endothelial cells with cytogenetic abnormalities. Cancer Res 64: 8249-8255, 2004.

19. Allport JR and Weissleder R: Murine Lewis lung carcinomaderived endothelium expresses markers of endothelial activation and requires tumor-specific extracellular matrix in vitro. Neoplasia 5: 205-217, 2003

20. Conaldi PG, Serra C, Mossa A, Falcone V, Basolo F, Camussi G, Dolei A and Toniolo A: Persistent infection of human vascular endothelial cells by group B coxsackieviruses. Infect Dis 175: 693-696, 1997.

21. Bussolati B, Biancone L, Cassoni P, Russo S, Montrucchio G, Rola-Pleszczynski $\mathrm{M}$ and Camussi G: PAF produced by human breast cancer cells promotes migration and proliferation of tumor cells and neo-angiogenesis. Am J Pathol 157: 1713-1725, 2000.

22. Kumar S, Ghellal A, Li C, Byrne G, Haboubi N, Wang J and Bundred N: Breast carcinoma: vascular density determined using CD105 antibody correlates with tumor prognosis. Cancer Res 59: 856-861, 1999.

23. Luscinskas FW and Lawler J: Integrins as dynamic regulators of vascular function. FASEB J 8: 929-938, 1994.

24. Huang HY, Ho CC, Huang PH and Hsu SM: Co-expression of VEGF-C and its receptors, VEGFR-2 and VEGFR-3, in endothelial cells of lymphangioma. Implication in autocrine or paracrine regulation of lymphangioma. Lab Invest 81: 1729-1734, 2001.
25. Partanen TA, Alitalo K and Miettinen M: Lack of lymphatic vascular specificity of vascular endothelial growth factor receptor 3 in 185 vascular tumors. Cancer 86: 2406-2412, 1999.

26. Kerbel RS: Clinical trials of antiangiogenic drugs: opportunities, problems, and assessment of initial results. J Clin Oncol 19: S45-S51, 2001.

27. Kerbel RS, Yu J, Tran J, Man S, Viloria-Petit A, Klement G, Coomber BL and Rak J: Possible mechanisms of acquired resistance to anti-angiogenic drugs: implications for the use of combination therapy approaches. Cancer Metastasis Rev 20: 79-86, 2001

28. Kurose K, Hoshaw-Woodard, S, Adeyinka A, Lemeshow S, Watson PH and Eng C: Genetic model of multi-step breast carcinogenesis involving the epithelium and stroma: clues to tumour-microenvironment interactions. Hum Mol Genet 10: 1907-1913, 2001.

29. Liu W, Ahmad SA, Reinmuth N, Shaheen RM, Jung YD, Fan F and Ellis LM: Endothelial cell survival and apoptosis in the tumor vasculature. Apoptosis 5: 323-328, 2000.

30. Flore O, Rafii S, Ely S, O'Leary JJ, Hyjek EM and Cesarman E: Transformation of primary human endothelial cells by Kaposi's sarcoma-associated herpesvirus. Nature 394: 588-592, 1998

31. Bagley RG, Walter-Yohrling J, Cao X, Weber W, Simons B, Cook BP, Chartrand SD, Wang C, Madden SL and Teicher BA: Endothelial precursor cells as a model of tumor endothelium: characterization and comparison with mature endothelial cells. Cancer Res 63: 5866-5873, 2003.

32. Hendrix MJ, Seftor EA, Kirschmann DA and Seftor RE: Molecular biology of breast cancer metastasis. Molecular expression of vascular markers by aggressive breast cancer cells. Breast Cancer Res 6: 417-422, 2000. 